\title{
Abusive Head Trauma in Infants and Children: Identifying Presenting Variables Associated With Outcome
}

Jessica L Carpenter, $M D^{1,2^{*}}$, Raquel Farias-Moeller, $M D^{1,3}$, Amy H Jones, $M D^{4,5}$, Shan Lateef ${ }^{1,6}$, Abigail Ley, $M D^{1,7}$ and Allison Jackson, $M D, M P H^{8}$

${ }^{1}$ Department of Neurology, Children's National Hospital, USA

${ }^{2}$ Departments of Neurology and Pediatrics, The George Washington University School of Medicine and Health Sciences, USA

${ }^{3}$ Department of Neurology and Pediatrics, Medical College of Wisconsin, Children's Wisconsin Milwaukee, USA

${ }^{4}$ Department of Critical Care Medicine, Children's National Hospital, USA

${ }^{5}$ Department of Pediatrics, The George Washington University School of Medicine and Health Sciences, USA

${ }^{6}$ Thomas Jefferson High School for Science and Technology, USA

${ }^{7}$ Department of Pediatrics, Child Neurology and Neurodevelopmental Disabilities, Florida State University, Fort Pierce, USA

${ }^{8}$ Department of Pediatrics, Division of Child Protection, Children's National Hospital, The George Washington University School of Medicine and Health Sciences, USA

\begin{abstract}
Objective: Correlate presenting clinical characteristics of abusive head trauma (AHT) patient with neurological outcome and late seizures.

Design: We review 52 cases of presumed abusive head trauma (AHT) in children $<24$ months of age admitted to a single center Pediatric Intensive Care Unit (PICU) between 2008 and 2017. Clinical variables were analyzed for association with neurological outcome $\geq 6$ months post-injury using the Glasgow outcome score-extended for pediatrics (GOS-E Peds) and late seizures. Only patients with $\geq 4$ hours of EEG within the first 72 hours of admission were included. Patients with coma and bilateral fixed and dilated pupils on presentation were excluded.
\end{abstract}

Results: Neurological outcome was assessed in 43 patients, of which 19 (44\%) had a poor outcome (GOS-E $\geq 5$ ). Cardiac arrest, GCS $\leq 8$ on presentation, parenchymal injury and longer stay in the PICU were identified as risk factors for poor outcome. Late seizures were present in $35 \%$ patients and were predicted by parenchymal injury but not acute symptomatic seizures.

Conclusions: Cardiac arrest and GCS $\leq 8$ were associated with poor outcome after AHT. Late seizures were not predicted by acute symptomatic seizures. Parenchymal injury was associated with both poor neurological outcome and late seizures.

\section{What's Known on This Topic}

- Children who suffer abusive head trauma are at high risk for long term neurological disability and epilepsy.

\section{What This Study Adds}

- Cardiac arrest on presentation and longer stay in the intensive care unit are associated with poor neurological outcome after AHT.

- Parenchymal injury on MRI is correlated with late seizures while acute symptomatic seizures are not.

Table of Contents Summary

Outcome after abusive head trauma is associated with presenting variables such as cardiac arrest and low GCS, as well as parenchymal injury on MRI. 


\begin{abstract}
Abbreviations
ADC: Apparent Diffusion Coefficient; AHT: Abusive Head Trauma; ASM: Anti-seizure medications; CAP: Child Abuse Pediatrics; Cl: Confidence Interval; CPR: Cardiopulmonary Resuscitation; CT: Computed Tomography; DWI: Diffusion Weighted Imaging; ED: Emergency Department; EEG: Electroencephalogram; EMR: Electronic Medical Record; EMS: Emergency Medical Services; EPTS: Early Post-Traumatic Seizures; GCS: Glasgow Coma Scale; GOS-E: Extended Glasgow Outcome Scale; HII: Hypoxic Ischemic Injury; ICP: Intracranial Pressure; IQR: Interquartile Range; MRI: Magnetic Resonance Imaging; NCC: Neurocritical Care; OR: Odds Ratio; PICU: Pediatric Intensive Care Unit; SES: Socioeconomic Status; SUDEP: Sudden Unexplained Death in an Epilepsy Patient
\end{abstract}

\section{Introduction}

Abusive head trauma (AHT) is a major cause of neurologic injury in young children with an estimated incidence of 12.8 to 17 per 100,000 in children < 2-years of age and 21 to 33.8 per 100,000 in children < 1-year [1]. The mortality rate can be as high as $36 \%$ [2] and survivors of AHT are subject to significant morbidity [3]. The majority of patients require long-term rehabilitation (up to $83 \%$ ) [4] and estimates of life time cost per single AHT patient are $>\$ 200,000$ [5-7].

Despite the high incidence of AHT, study of these patients is largely limited to descriptors of small cohorts identified retrospectively. Age at the time of inflicted injury and presenting Glasgow Coma Score (GCS) are reported as variables predictive of poor outcome at discharge [8]. Acute symptomatic seizures occur in up to $57 \%$ of AHT patients [5] and are often non-convulsive [9]. While many studies have investigated the incidence and characteristics of early posttraumatic seizures $[9,10]$, far less is known about the presenting variables associated with late seizures which are common after AHT. Predictors for developing epilepsy remain poorly defined.

Given the complexities of care associated with AHT, a multidisciplinary approach to the evaluation and management of these patients is critical [11]. Over the last decade, pediatric neurocritical care (NCC) programs specializing in the care of critically ill children with acute brain injury have become more common [12,13]. Many hospitals also have access to child abuse pediatricians (CAPs), who specialize in the evaluation of children who may be victims of abuse or neglect. When NCC teams combine with CAPs, they collectively provide AHT patients with a high level of care by clinicians who are familiar with the intricacies of AHT. Historically, AHT patients were subject to heterogeneity of evaluation and management, factors which may contribute to outcome differences. We performed a review of presumed AHT patients prospectively identified and cared for in a standardized fashion. We aimed to investigate patient characteristics and clinical variables associated with poor long term neurological outcome. Risk factors for the development of late seizures were assessed as a secondary outcome measure.

\section{Study Design}

Children with a diagnosis of presumed AHT admitted to a single tertiary care Pediatric Intensive Care Unit (PICU) between 2008 and 2017 were analyzed. Patients were identified from a NCC database. Additional clinical variables were retrospectively ascertained through review of the electronic medical record (EMR), including records from referring facil- ities and Emergency Medical Services (EMS). The study was approved by Children's National Hospital Institutional Review Board, Pro 00001916.

Consecutive patients $<24$ months of age with presumed AHT were included in the analysis. The diagnosis of presumed AHT was determined by our child protection team after comprehensive clinical consultation and multidisciplinary input. Standard institutional practice is for patients with a new diagnosis of intracranial hemorrhage to be initially admitted to the PICU independent of mental status at the time of presentation. All patients were evaluated by the NCC team within 24 hours of presentation and subject to institutional AHT management recommendations (Supplement 1). Corrected gestation age was used for patients with a history of prematurity. Only patients with at least 4 hours of continuous electroencephalogram (EEG) monitoring during the first 72 hours of admission were included. Patients with coma associated with bilateral fixed and dilated pupils on presentation were excluded due to the concern of imminent mortality.

\section{Clinical characteristics}

Patient characteristics assessed for impact on functional outcome included gender, age, prematurity and SES. Presenting factors, including site of presentation (i.e. regional vs. tertiary ED), initial GCS, retinal hemorrhage, extra-cranial fractures and cardiac arrest were also evaluated as potential risk factors for severe neurological disability. Absent or public insurance at the time of presentation was used as a surrogate marker of low SES. Site of presentation was captured as tertiary care versus non-tertiary care center. Presenting GCS was captured as the first pediatric GCS assigned by EMS. In the absence of EMS documents, the first GCS recorded at the presenting ED were used. GCS $\leq 8$ was used as a marker of severe injury, per TBI convention [14]. All AHT patients were evaluated by a pediatric ophthalmologist as per institutional practice. Only retinal hemorrhages diagnosed and described by an ophthalmologist were considered. Finally, only children

*Corresponding author: Jessica L Carpenter, MD, Department of Child Neurology, Children's National Hospital, 111 Michigan Avenue, Washington DC 20010, USA, Tel: 202-476-2120

Accepted: June 02, 2021

Published online: June 04, 2021

Citation: Carpenter JL, Moeller RF, Jones AH, et al. (2021) Abusive Head Trauma in Infants and Children: Identifying Presenting Variables Associated With Outcome. J Pediatr Neurol Neurosci 5(1):110-118 
Citation: Carpenter JL, Moeller RF, Jones AH, et al. (2021) Abusive Head Trauma in Infants and Children: Identifying Presenting Variables Associated With Outcome. J Pediatr Neurol Neurosci 5(1):110-118

who received cardiopulmonary resuscitation (CPR) by either EMS or hospital staff were documented as having a cardiac arrest on presentation. Length of PICU stay and total hospital stay were calculated and included in the analysis.

\section{Radiographic characteristics}

Intracranial injuries were diagnosed through a combination of head computed tomography scan (CT) and magnetic resonance imaging (MRI) of the brain. The presence of intracranial hemorrhage, parenchymal injury and/or skull fracture was noted. Parenchymal injury was defined as abnormalities of the cerebrum seen on MRI, including intra parenchymal hemorrhage, contusion or ischemia. The clinical report generated by a pediatric neuroradiologist was used to establish the radiographic findings. For any report with unclear or ambiguous language, images were reviewed by a pediatric neurologist to define the injuries.

\section{Clinical and electrographic seizures}

Per institutional routine, all children with intracranial hemorrhage and GCS $<12$ are monitored with continuous EEG. Due to the high risk of seizures associated with AHT, it is recommended that all patients with presumed AHT be monitored independent of altered consciousness. While 24 hours of monitoring is recommended, in practice, the duration of monitoring varies based on the patient's stability, physician practice and resource allocation. All EEGs were obtained with a standard or modified 10-20 montage.

Seizure on presentation was defined as any seizure occurring in the field, EMS transport or emergency department (ED). Events were included only if there was a clear clinical description documented where the most likely explanation of the event was a clinical seizure. Events that could not be distinguished from posturing, focal neurological deficits (e.g. gaze palsy) and/or altered consciousness were not captured as seizure on presentation. Acute symptomatic seizure was defined as any clinical or electrographic seizure occurring within 7 days of the AHT. Seizures on presentation were included as acute symptomatic seizures. All continuous infusion medications with anti-seizure properties were evaluated for intent of use (e.g. sedation vs. seizure treatment).

\section{Outcome measures}

Neurological outcome was measured utilizing the Glasgow Outcome Scale - Extended for pediatrics (GOS-E Ped). The GOS-E Ped is validated in young children and is composed of eight categories ranging from normal function (1) to death (8) [15]. GOS-E Ped scores were assigned based on the neurology, neurosurgery and/or physical medicine and rehabilitation clinic notes. Scores were assigned $\geq 6$ months post-injury and determined to be favorable if the GOS-E was $<5$ ( $4=$ lower moderate disability) and unfavorable if the GOS-E was $\geq 5$ ( 5 = upper severe disability). When multiple clinic notes existed for a given patient, the most recent was used to allow for the longest possible recovery.

Incidence of late seizures was used as a secondary outcome measure. Late seizure was defined as any seizure occurring $>7$ days after the acute brain insult. The day of the insult was reported as the day of presentation for all patients. Both inpatient and outpatient documents were used to assess for the presence of late seizures.

\section{Data analysis}

Descriptive statistics were used to identify differences in clinical characteristics and elements of the clinical course among patients with and without poor neurologic outcome as well as those with or without late onset seizures.

For categorical variables, comparisons were made using Fisher exact test; for continuous variables, the Mann-Whitney $U$ test was used. The candidate predictors with a significance level of $p<0.5$ were identified by univariate analysis. For the categorical variables significant in univariate analyses, odds ratios were calculated using the Fisher exact test as our small sample size did not permit logistic regression analyses. Post-hoc false discovery rate testing was conducted using the Benjamini-Hochberg procedure to adjust for multiple comparisons, and a False Discovery Rate of 0.1 was used. All univariate analyses were conducted using XLSTAT. Odds Ratios (OR) were generated using Med Calc Statistical Calculator (Medcalc.org, 2019).

\section{Results}

\section{Demographics}

A total of 82 patients with presumed AHT were identified, of which 52 met inclusion criteria (Figure 1). Eighteen presented with coma associated with bilateral fixed and dilated pupils and thus were excluded from the analysis. All but one of these patients suffered inpatient mortality. Twelve patients were excluded due to insufficient EEG monitoring.

Patient characteristics are summarized in Table 1 . The median age at presentation was 3.7 months (range 0.6 -20 months corrected gestational age). Twelve patients had a history of prematurity (23\%, range $26-36$ weeks estimated gestational age). A majority of patients (75\%) were estimated to be of low SES based on insurance type.

\section{Clinical presentation}

Thirty-nine patients (75\%) presented initially to a local hospital prior to transfer to our tertiary care center. One patient was not included in the GCS analysis because the records from the outside hospital were not available for review. Less than half of patients had an initial GCS $\leq 8$ (45\%, $n=$ $23 / 51)$, of which eight had a GCS of 3 . Retinal hemorrhages were present in all but 7 patients $(87 \%, n=45 / 52)$. There were 7 patients with cardiac arrest (13\%).

\section{Radiological findings}

All patients had at least one head CT and one brain MRI. All had some degree of subdural hemorrhage. Thirty-nine patients $(75 \%, n=39 / 52)$ had parenchymal injury. Nine patients suffered a skull fracture and twenty-one had extra cranial fractures.

\section{Seizures}

The overall incidence of symptomatic seizures was $75 \%$ 
Citation: Carpenter JL, Moeller RF, Jones AH, et al. (2021) Abusive Head Trauma in Infants and Children: Identifying Presenting Variables Associated With Outcome. J Pediatr Neurol Neurosci 5(1):110-118

Patients with presumed AHT $(n=82)$

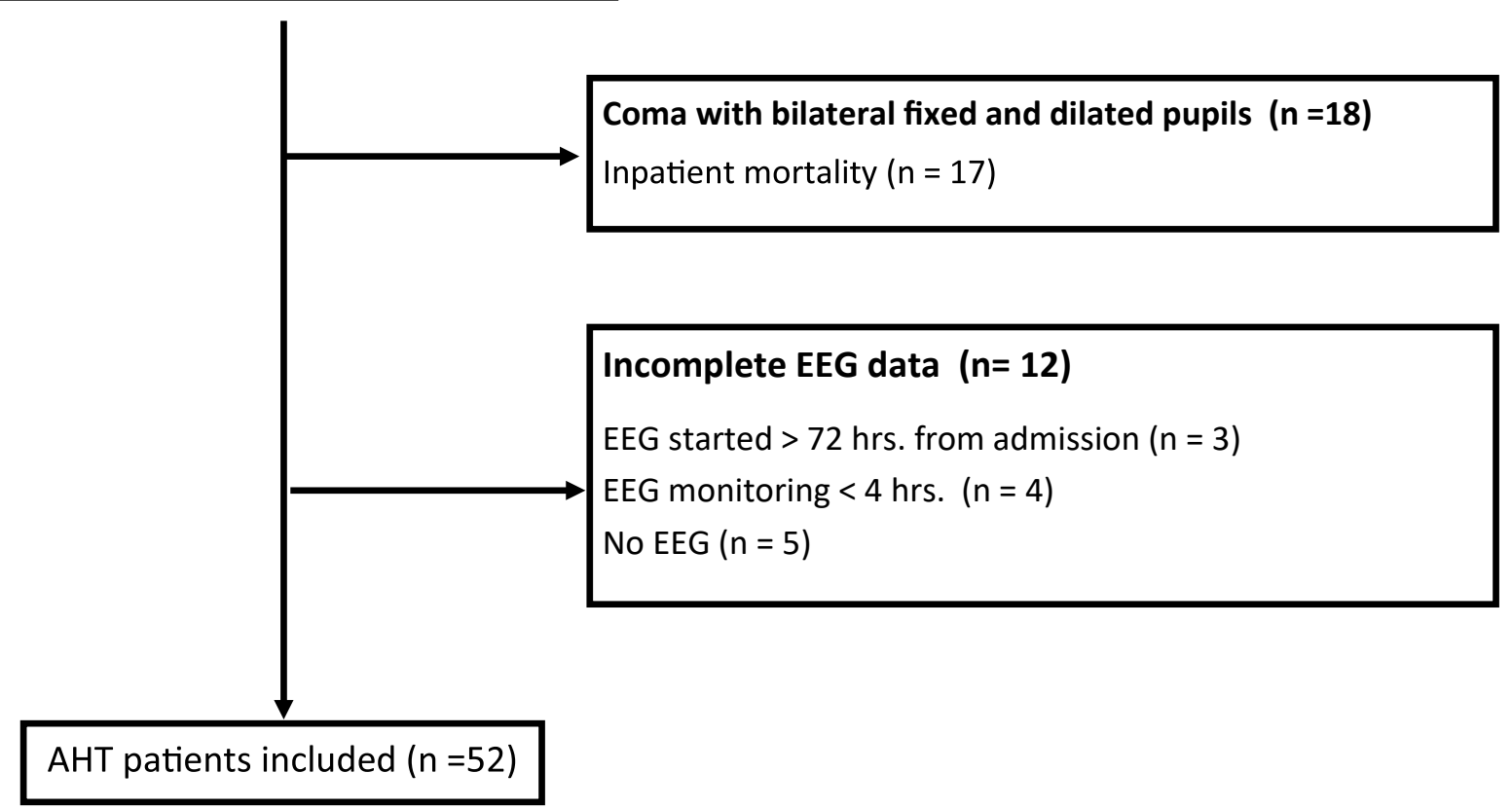

Figure 1: Flowchart of patient selection.

Table 1: Demographics and clinical characteristics (patients excluded due to insufficient EEG monitoring presented for comparison).

\begin{tabular}{|c|c|c|}
\hline & $\begin{array}{l}\text { Presumed AHT patients with inclusion \& } \\
\text { exclusion criteria met } \\
(n=52)\end{array}$ & $\begin{array}{l}\text { Presumed AHT patients excluded for } \\
\text { insufficient EEG monitoring } \\
(n=12)\end{array}$ \\
\hline \multicolumn{3}{|l|}{ Patient Characteristics } \\
\hline Male, n (\%) & $35(67)$ & $8(67)$ \\
\hline Age at presentation, median $(I Q R)^{f}$ & $3.7(2.1-5.4)$ & $4.1(3-9.5)$ \\
\hline Prematurity, n (\%) & $12(23)$ & $3(25)$ \\
\hline Insurance type - Medicaid & $39(75)$ & $9(75)$ \\
\hline \multicolumn{3}{|l|}{ Clinical course } \\
\hline Initial presentation to OSH, $\mathrm{n}(\%)$ & $39(75)$ & $7(58)$ \\
\hline Cardiac arrest, n (\%) & $7(13)$ & 0 \\
\hline Patients with acute $\mathrm{HCT}$ and brain MRI & $52(100)$ & $12(100)$ \\
\hline MRI with parenchymal injury, $\mathrm{n}(\%)$ & $39(75)$ & $4(33)$ \\
\hline Inpatient mortality, n (\%) & $3(6)$ & 0 \\
\hline Days of ICU admission, median (IQR) & $7(3-10)$ & $3(1.8-3.3)$ \\
\hline Hospital length of stay, median (IQR) & $14(8-20)$ & $7(5-13.3)$ \\
\hline Year of admission, median (IQR) & $2012(2010-2015)$ & 2012 (2009 -2012) \\
\hline Follow up $\geq 6$ months post injury, $\mathrm{n}(\%)$ & $43(83)$ & $8(67)$ \\
\hline
\end{tabular}

${ }^{\mathrm{f} C o r r e c t e d ~ f o r ~ g e s t a t i o n a l ~ a g e ~(m e a s u r e d ~ i n ~ m o n t h s) ; ~}{ }^{*} \mathrm{n}=51$ (one patient did not have a peds GCS assigned)

( $n=39 / 52)$ with $56 \%(n=29 / 52)$ having seizures on presentation. Of patients with symptomatic seizures, $28 \%(11 / 40)$ were documented to have their first seizure after admission to the PICU. Thirty eight percent (20/52) had electrographic seizures. Only one patient had non convulsive seizures without a clinical seizure. Fourteen children had refractory seizures requiring treatment with a continuous infusion of an anti-seizure medication (ASM). Twelve patients (23\%) received an infusion of a medication with anti-seizure properties for the intended use of sedation; three of which had the infusion up-titrated for the purpose of seizure control once seizures were identified. Patients with symptomatic seizures 
Citation: Carpenter JL, Moeller RF, Jones AH, et al. (2021) Abusive Head Trauma in Infants and Children: Identifying Presenting Variables Associated With Outcome. J Pediatr Neurol Neurosci 5(1):110-118

Table 2: Patient characteristics and clinical variables associated with poor neurological outcome and late seizures.

\begin{tabular}{|c|c|c|c|c|c|c|c|}
\hline \multirow[b]{2}{*}{ Patient Characteristics } & \multicolumn{4}{|c|}{$\begin{array}{l}\text { GOS-E Assessed at Least } 6 \text { months Post Injury } \\
(n=52)\end{array}$} & \multicolumn{3}{|c|}{$\begin{array}{l}\text { Persistent Seizures After } 7 \text { days Post Injury } \\
(n=51)\end{array}$} \\
\hline & $\begin{array}{l}\text { No GOS-E } \\
n=9\end{array}$ & GOS-E $\leq 5 \mathrm{n}(\%)$ & $\begin{array}{l}\text { GOS-E > } 5 \\
\mathrm{n}(\%)\end{array}$ & P-Value & $\begin{array}{l}\text { Late seizures Absent } \\
\mathrm{n}(\%)\end{array}$ & $\begin{array}{l}\text { Late seizures } \\
\text { Present } \mathrm{n}(\%)\end{array}$ & P-Value \\
\hline \multicolumn{8}{|l|}{ Gender } \\
\hline Male & 6 & $18(62.1)$ & 11 (37.9) & 0.329 & $24(70.6)$ & $10(29.4)$ & 0.531 \\
\hline Female & 3 & $6(42.9)$ & $8(57.1)$ & & $10(58.8)$ & $7(41.2)$ & \\
\hline Mean age & 5.4 & 4.6 & 5 & 0.774 & 4.2 & 6.3 & 0.119 \\
\hline \multicolumn{8}{|l|}{ Prematurity } \\
\hline No & 7 & $20(60.6)$ & $13(39.4)$ & 0.295 & $26(65.0)$ & $14(35)$ & 0.731 \\
\hline Yes & 2 & $4(40.0)$ & $6(60.0)$ & & 8 (72.7) & $3(27.3)$ & \\
\hline \multicolumn{8}{|l|}{ Insurance type } \\
\hline Private & 2 & $5(45.4)$ & $6(54.6)$ & 0.495 & $7(53.8)$ & $6(46.2)$ & 0.315 \\
\hline Medicaid/None & 7 & $19(59.4)$ & $13(40.6)$ & & $27(71.1)$ & $11(28.9)$ & \\
\hline \multicolumn{8}{|l|}{ Presenting factors } \\
\hline \multicolumn{8}{|l|}{ Outside Hospital } \\
\hline No & 2 & $6(54.6)$ & $5(45.4)$ & 0.995 & $9(69.2)$ & $4(30.7)$ & 1 \\
\hline Yes & 7 & $18(56.3)$ & $14(43.7)$ & & $25(65.8)$ & $13(34.2)$ & \\
\hline \multicolumn{8}{|l|}{ Glasgow Coma Scale } \\
\hline $\begin{array}{l}\text { GCS }>8 \text { (mild to moderate } \\
\text { TBI) }\end{array}$ & 9 & $14(73.7)$ & $5(26.3)$ & 0.033 & $20(74.1)$ & 7 (25.9) & 0.228 \\
\hline $\mathrm{GCS} \leq$ (severe $\mathrm{TBI})$ & 0 & 9 (39.1) & $14(60.9)$ & & $13(56.5)$ & $10(43.5)$ & \\
\hline \multicolumn{8}{|l|}{ Retinal hemorrhages $(\mathrm{Y} / \mathrm{N})$} \\
\hline No & 1 & $6(100.0)$ & $0(0)$ & 0.027 & $6(85.7)$ & $1(14.3)$ & 0.401 \\
\hline Yes & 8 & $18(48.7)$ & $19(51.3)$ & & $28(77.8)$ & $16(28.2)$ & \\
\hline \multicolumn{8}{|l|}{ Cardiac arrest } \\
\hline No & 9 & $24(66.7)$ & $12(33.3)$ & 0.002 & $31(68.9)$ & $14(31.1)$ & 0.387 \\
\hline Yes & 0 & $0(0)$ & $7(100.0)$ & & $3(50.0)$ & $3(50.0)$ & \\
\hline \multicolumn{8}{|l|}{ Radiology } \\
\hline \multicolumn{8}{|l|}{ Parenchymal injury } \\
\hline No & 4 & $9(100)$ & $0(0)$ & 0.002 & $12(92.3)$ & $1(7.7)$ & 0.038 \\
\hline Yes & 5 & $15(44.9)$ & $19(55.1)$ & & $22(57.9)$ & $16(42.1)$ & \\
\hline \multicolumn{8}{|l|}{ Skull fracture } \\
\hline No & 7 & $22(61.1)$ & $14(38.9)$ & 0.211 & $28(66.7)$ & $14(33.3)$ & 1 \\
\hline Yes & 2 & $2(28.6)$ & $5(71.4)$ & & $6(66.7)$ & $3(33.3)$ & \\
\hline \multicolumn{8}{|l|}{ Other fractures } \\
\hline No & 5 & $15(57.7)$ & $11(42.3)$ & 1 & & & \\
\hline Yes & 4 & 9 (52.9) & $8(47.1)$ & & & & \\
\hline \multicolumn{8}{|l|}{ Seizures } \\
\hline \multicolumn{8}{|l|}{ Seizures on presentation } \\
\hline No & 2 & $10(47.6)$ & $11(52.4)$ & 0.364 & $13(59.1)$ & 9 (40.9) & 0.377 \\
\hline Yes & 7 & $14(63.6)$ & $8(36.4)$ & & $21(72.4)$ & $8(27.6)$ & \\
\hline \multicolumn{8}{|c|}{ Seizure within 7 days of injury } \\
\hline No & 2 & $4(40.0)$ & $6(60.0)$ & 0.295 & $8(72.7)$ & $3(27.3)$ & 0.731 \\
\hline Yes & 7 & $20(60.6)$ & $13(39.4)$ & & $26(65.0)$ & $14(35.0)$ & \\
\hline
\end{tabular}


Citation: Carpenter JL, Moeller RF, Jones AH, et al. (2021) Abusive Head Trauma in Infants and Children: Identifying Presenting Variables Associated With Outcome. J Pediatr Neurol Neurosci 5(1):110-118

\begin{tabular}{|c|c|c|c|c|c|c|c|}
\hline \multicolumn{8}{|c|}{ Seizures treated with infusion } \\
\hline No & 7 & $21(63.6)$ & $10(36.4)$ & 0.017 & $27(73.0)$ & $10(27.0)$ & 0.183 \\
\hline Yes & 2 & $3(25.0)$ & $9(75)$ & & $7(50.0)$ & $7(50.0)$ & \\
\hline \multicolumn{8}{|l|}{ Clinical course } \\
\hline Average length of ICU stay & 3.4 & 6.2 & 14 & 0.001 & 7.2 & 11.6 & 0.025 \\
\hline $\begin{array}{l}\text { Average Length of hospital } \\
\text { stay }\end{array}$ & 8.9 & 12.8 & 22.8 & 0.005 & 13.9 & 20.5 & 0.01 \\
\hline
\end{tabular}

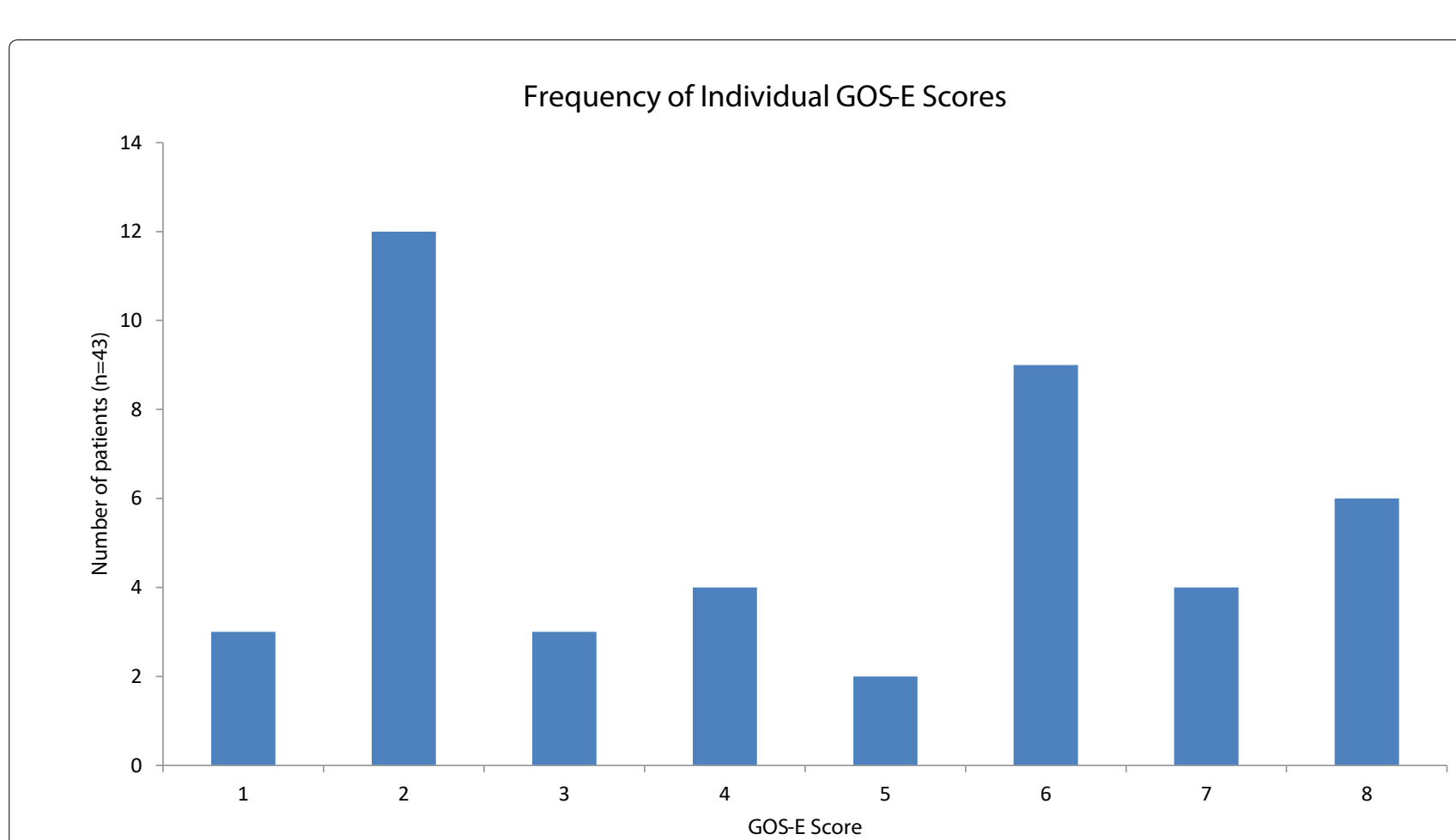

Figure 2: Individual GOS-E scores assigned $\geq 6$ months post injury.

had a median age of 2.9 months (inter quartile range (IQR) 2-4 months) compared to 6.7 months (IQR 5-12 months) for those without seizures. No child in the study had a pre-existing diagnosis of epilepsy.

For the patients who survived beyond seven days from presentation, late seizures occurred in 17 (33\%, 17/51). Only one patient developed late seizures during the initial hospitalization, the rest had their first late seizure in the outpatient setting. The median follow up for assessment of late seizures was 28 months (IQR8 to 58 months). Parenchymal injury and longer PICU stay were correlated with an increased risk for late seizures ( $p=0.038$ and 0.025 , respectively). Symptomatic seizures did not predict late seizures, nor did refractory seizures treated with infusion medications (Table 2). Of the 49 patients who survived to discharge, $44(90 \%)$ continued to receive an ASM at discharge and $22(45 \%)$ were prescribed an $\mathrm{AMS}$ at the most recent follow up.

\section{Hospital course}

Length of PICU stay and total length of hospital admission were highly varied. Median duration of intensive care admission was 7 days (range 1-42) and median duration of total hospital stay was 14 days (range 3-54 days). Only 3 pa- tients suffered inpatient mortality. An additional 3 died after discharge, for a total mortality of $12 \%$.

\section{Neurological outcome}

Forty-three patients were assigned $a \geq 6$ month post-injury GOS-E Ped score. Outcomes were highly varied (Figure 2). Follow up assessments were performed on visits a median of 40 months after injury (range 0-130 months, Supplement 2). Twenty-four patients had a favorable neurologic outcome (GOS-E $\leq 5)$ and 19 had severe neurological disability or death (GOS-E $>5$ ). Six died ( 5 deaths occurred $<6$ months post injury). One died $>3$-years after the insult, likely from sudden unexplained death in an epilepsy patient (SUDEP). Variables predictive of a poor outcome were cardiac arrest $(p=0.002)$, presenting GCS $\leq 8(p=0.033)$, retinal hemorrhages $(p=0.027)$ parenchymal injury $(p=0.002)$, refractory seizures requiring treatment with an infusion ( $p=0.017)$, length of PICU stay $(p=0.001)$ and duration of total hospital course $(p=0.005)$ (Table 3). SES was not predictive of poor outcome.

\section{Discussion}

We describe a single center, case series of AHT patients cared for by an NCC team in a standardized fashion and re- 
Citation: Carpenter JL, Moeller RF, Jones AH, et al. (2021) Abusive Head Trauma in Infants and Children: Identifying Presenting Variables Associated With Outcome. J Pediatr Neurol Neurosci 5(1):110-118

Table 3: Predictors of Poor Neurologic Outcome after Abusive Head Trauma.

\begin{tabular}{|l|l|l|l|}
\hline \multirow{2}{*}{} & \multicolumn{3}{|c|}{ Predictors of Poor GOS-E } \\
\cline { 2 - 4 } & OR & $\mathbf{9 5 \%}$ Cl & p-Value \\
\hline $\begin{array}{l}\text { Seizures Treated with } \\
\text { Infusion }\end{array}$ & 6.3 & $1.39-28.47$ & $\mathbf{0 . 0 1 7}$ \\
\hline Cardiac Arrest & 29.6 & $1.6-557.6$ & $\mathbf{0 . 0 2 4}$ \\
\hline GCS </= 8 & 4.36 & $1.16-16.31$ & $\mathbf{0 . 0 2 9}$ \\
\hline Parenchymal Brain Injury & 23.9 & $1.29-443.65$ & $\mathbf{0 . 0 3 3}$ \\
\hline Retinal Hemorrhage & 13.7 & $0.72-260.7$ & 0.082 \\
\hline
\end{tabular}

port factors associated with poor neurologic outcome and late seizures. Cardiac arrest, initial GCS, retinal hemorrhage, parenchymal brain injury and refractory seizures necessitating treatment with a continuous infusion medication were all associated with unfavorable neurologic outcome $\geq 6$ months after injury. Children with unfavorable neurologic outcome also had longer hospitalizations and more prolonged PICU stays. Late seizures were associated with parenchymal injury as well as longer hospitalization.

The distribution of ages in this cohort is similar to prior publications reporting on the epidemiology of inflicted trauma $[2,6,16]$. Neither age nor prematurity influenced neurological outcome. This is dissimilar to Chen, et al.'s finding of older age as an independent risk factor for poor outcome, though their study included patients up to 4 years of age [8].

A majority of the patients $(75 \%)$ in this study were estimated to be of low SES, similar to other studies $[6,16,17]$. In this series, SES was not correlated with a poor outcome as previously reported $[4,18]$. Likely because our analysis was under powered as a product of the small number with private insurance.

Retinal hemorrhages were present in $87 \%$ of patients, also consistent with the AHT literature. Extensive retinal hemorrhages are reported to be associated with worse neurologic outcomes and are theorized to be reflective of a more violent infliction of trauma [19]. While we did not evaluate the extent of retinal hemorrhage, our study suggests the presence of retinal hemorrhage itself may be a hallmark for worse outcome.

In this series, presenting GCS $(\leq 8)$ was also predictive of poor long term outcome, similar to other AHT studies reporting poor outcome at discharge [8]. While a minority of patients received CPR $(n=7)$, the effect on outcome was consistent and profound. All seven patients either died or were profoundly disabled (GOS-E 7 or 8). The negative impact of hypoxic ischemic injury (HII) is previously established. Ichord, et al. [20] reported AHT patients with HII by MRI had an increased need for inpatient rehabilitation compared to those without.

One of the strengths of this study is the uniformity of imaging obtained. All patients, consecutively captured, had at least one head CT and brain MRI. Median time from admission to brain MRI was 1.5 days (IQR 1.0-3.3) and 12 patients had multiple MRIs. Subdural hemorrhages were universal, likely due to the established clinical guidelines to diagnose
AHT [21]. Parenchymal injury was described in $75 \%$, which is similar to other reports of AHT including MRI imaging [22-25]. Based on this analysis, identification of parenchymal injury is critical not only because it is associated with poor neurological outcomes, but because it is also predictive of late seizure development.

A high rate of symptomatic seizures (75\%) was present in this cohort, consistent with other investigations [25-28]. All but one patient had a clinical seizure prior to developing electrographic seizures. This low rate of electrographic-only seizures is significant because it demonstrates the AHT patients most likely to benefit from EEG monitoring for the detection of non-convulsive seizures are those with a preceding clinical event. In this study, "seizures on presentation" were characterized as symptomatic seizures. This is distinct from some authors who define seizure on presentation as "impact seizures" and seizures occurring within the first week after injury but not immediately after impact as "early post-traumatic seizures (EPTS)" [28].

The close correlation between HII and seizure burden in AHT patients, established by Dingman, et al., highlights the importance of both EEG monitoring and MR imaging to guide acute management in this population [25]. Our analysis also suggests seizures on presentation and neuroimaging with HII are presenting features which distinguish patients in greatest need of EEG monitoring.

Thirty five percent of patients who survived beyond 7 days ( $n=17 / 51$ ) experienced late seizures, which is similar to other studies describing the incidence of epilepsy after AHT $[4,29]$ Parenchymal injury, but not acute symptomatic seizures, was correlated with late seizures.

The duration of PICU stay was highly varied in this cohort. The analysis demonstrated an association of longer admission with worse functional outcome. While it is generally accepted that patients with more severe disease have longer hospital courses due to the complexity of their disease, it is also increasingly recognized that longer PICU stays may contribute to morbidity of patients with acute brain injury $[30,31]$.

Several studies have reported on neurological outcome after AHT $[4,8,29]$. This cohort is unique in that we are able to describe long term outcomes in a cohort of consecutive AHT patients identified prospectively and treated with relative uniformity. Similar to the existing literature, a high rate of neurological disability and death was noted. Less well described is the full spectrum of outcomes noted (Figure 2).

In 2016, Lind, et al, reported on long term outcomes of a cohort of AHT patients identified from a single rehabilitation center [4]. They also noted a high incidence of severe neurological disability (40\%, median follow up 8-years after injury) by GOS. Despite the difference in scoring systems used, outcomes were similar. In our cohort, $44 \%$ of patients suffered severe disability or death.

More recently, a large series of AHT patients $(n=940)$ identified from a Truven Health Market Scan Research Data base also reported poor long-term outcomes [29]. This publication emphasized that although developmental delay/intel- 
Citation: Carpenter JL, Moeller RF, Jones AH, et al. (2021) Abusive Head Trauma in Infants and Children: Identifying Presenting Variables Associated With Outcome. J Pediatr Neurol Neurosci 5(1):110-118

lectual disability, motor deficits, and epilepsy may be recognized at early follow up (3-9 months post-injury), factors such as behavioral disorders, communication deficits and visual impairment are often not recognized until much later (2446 months post-injury). In our series, excluding patients who died, the median GOS-EP eds score was assigned at 43months (range 6-130 months) post-injury.

Our case series has several limitations. The small sample size limited our ability to perform multivariate analyses. The 12 patients without sufficient EEG monitoring that were excluded represented milder forms of AHT (Table 1), and by not including them we introduced a selection bias. The indirect measure (i.e. Medicaid coverage) of low SES contributed to an under representation of children living in low income households. Nine patients were not eligible for outcome analysis because they were lost to follow up. None of these patients suffered a cardiac arrest and all had a presenting GCS $>8$. These patients collectively also likely represent individuals with milder forms of AHT. Finally, because children with coma associated with bilateral fixed and dilated pupils were excluded from the analysis the mortality rate is underestimated (mortality for all AHT patients identified was $28 \%$, $23 / 83$ ). Finally, the follow up range selected was broad. This approach was taken to allow for maximal recovery and the most accurate assessment possible using the outcome scores selected. By taking this approach, we sacrificed uniformity in the recovery interval.

\section{Conclusion}

This study reinforces that AHT is associated with a high risk for poor long-term neurological outcome (44\%), and late seizures (33\%). Retinal hemorrhages, presenting GCS $\leq 8$ and cardiac arrest were associated with poor neurologic outcome. Parenchymal injury was the strongest predictor of outcome and was associated with both poor neurologic outcome and late symptomatic seizures.

\section{Funding Source}

No external funding for this manuscript.

\section{Financial Disclosure}

The authors have indicated they have no financial relationships relevant to this article to disclose.

\section{Conflict of Interest}

The authors have no potential conflicts of interest to disclose.

\section{References}

1. Cowley LE, Morris CB, Maguire SA, et al. (2015) Validation of a prediction tool for abusive head trauma. Pediatrics 136: 290298.

2. Thompson LW, Bass KD, Agyei JO, et al. (2019) Incidence of nonaccidental head trauma in infants: $A$ call to revisit prevention strategies. J Neurosurg Pediatr 20: 1-8.

3. King WJ, MacKay M, Sirnick A (2003) Shaken baby syndrome in Canada: Clinical characteristics and outcomes of hospital cases. Cmaj 168: 155-159.
4. Lind $\mathrm{K}$, Toure $\mathrm{H}$, Brugel D, et al. (2016) Extended follow-up of neurological, cognitive, behavioral, and academic outcomes after severe abusive head trauma. Child Abuse \& Negl 51: 358-367.

5. Fang X, Brown DS, Florence CS, et al. (2012) The economic burden of child maltreatment in the United States and implications for prevention. Child Abuse \& Neglect 36: 156-165.

6. Boop S, Axente M, Weatherford B, et al. (2016) Abusive head trauma: An epidemiological and cost analysis. J Neurosurg Pediatr 18: 542-549.

7. Peterson C, Xu L, Florence C, et al. (2015) Annual cost of US hospital visits for pediatric abusive head trauma. Child Maltreat 20: 162-169.

8. Chen CC, Hsieh PC, Chen CP, et al. (2019) Prevention PA. Clinical characteristics and predictors of poor hospital discharge outcome for young children with abusive head trauma. J Clin Med 8: 390.

9. Oh A, Olson LD, Chern JJ, et al. (2019) Clinical characteristics and nonconvulsive seizures in young children with abusive head trauma. J Child Neurol 34: 713-719.

10. O'Neill BR, Handler MH, Tong S, et al. (2015) Incidence of seizures on continuous EEG monitoring following traumatic brain injury in children. J Neurosurg Pediatr 16: 167-176.

11. Choudhary AK, Servaes S, Slovis TL, et al. (2018) Consensus statement on abusive head trauma in infants and young children. Pediatr Radiol 48: 1048-1065.

12. Bell MJ, Carpenter J, Au AK, et al. (2009) Development of a pediatric neurocritical care service. Neurocrit Care 10: 4-10.

13. LaRovere KL, Graham RJ, Tasker RC (2013) Pediatric Critical Nervous System Program ( $\mathrm{CCNSp}$. Pediatric neurocritical care: A neurology consultation model and implication for education and training. Pediatr Neurol 48: 206-211.

14. Bullock RC, Chesnut RM, Clifton G, et al. (1996) Guidelines for the management of severe head injury. Eur J Emerg Med 3: 109127.

15. Beers SR, Wisniewski SR, Garcia-Filion P, et al. (2012) Validity of a pediatric version of the Glasgow outcome scale-extended. J Neurotrauma 29: 1126-1139.

16. Niederkrotenthaler T, Xu L, Parks SE, et al. (2013) Descriptive factors of abusive head trauma in young children-United States, 2000-2009. Child Abuse Negl 37: 446-455.

17. Selassie AW, Borg K, Busch C, et al. (2013) Abusive head trauma in young children: A population-based study. Pediatric Emergency Care 29: 283-291.

18. Nuño M, Pelissier L, Varshneya K, et al. (2015) Outcomes and factors associated with infant abusive head trauma in the US. J Neurosurg Pediatr 16: 515-522.

19. Binenbaum G, Christian CW, Ichord RN, et al. (2013) Retinal hemorrhage and brain injury patterns on diffusion-weighted magnetic resonance imaging in children with head trauma. J AAPOS 17: 603-608.

20. Ichord RN, Naim M, Pollock AN, et al. (2007) Hypoxic-ischemic injury complicates inflicted and accidental traumatic brain injury in young children: The role of diffusion-weighted imaging. J Neurotrauma 24 : $106-118$.

21. Christian CW, Committee on Child Abuse and Neglect (2015) The evaluation of suspected child physical abuse. Pediatrics 135: e1337-e1354. 
Citation: Carpenter JL, Moeller RF, Jones AH, et al. (2021) Abusive Head Trauma in Infants and Children: Identifying Presenting Variables Associated With Outcome. J Pediatr Neurol Neurosci 5(1):110-118

22. Foerster BR, Petrou M, Lin D, et al. (2009) Neuroimaging evaluation of non-accidental head trauma with correlation to clinical outcomes: A review of 57 cases. J Pediatr 154: 573-577.

23. Vázquez E, Delgado I, Sánchez-Montañez A, et al. (2014) Imaging abusive head trauma: why use both computed tomography and magnetic resonance imaging?. Pediatr Radiol 44: s589-s603.

24. Goldstein JL, Leonhardt D, Kmytyuk N, et al. (2011) Abnormal neuroimaging is associated with early in-hospital seizures in pediatric abusive head trauma. Neurocrit Care 15: 63-69.

25. Dingman AL, Stence NV, O'Neill BR, et al. (2018) Seizure severity is correlated with severity of hypoxic-ischemic injury in abusive head trauma. Pediatr Neurol 82: 29-35.

26. Barlow KM, Spowart JJ, Minns RA (2000) Early posttraumatic seizures in non-accidental head injury: Relation to outcome. Dev Med Child Neurol 42: 591-594.
27. Arndt DH, Lerner JT, Matsumoto JH, et al. (2013) Subclinical early posttraumatic seizures detected by continuous EEG monitoring in a consecutive pediatric cohort. Epilepsia 54: 1780-1788.

28. Liesemer K, Bratton SL, Zebrack CM, et al. (2011) Early post-traumatic seizures in moderate to severe pediatric traumatic brain injury: Rates, risk factors, and clinical features. J Neurotrauma 28: 755-762.

29. Nuño M, Ugiliweneza B, Zepeda V, et al. (2018) Long-term impact of abusive head trauma in young children. Child Abuse Negl 85: 39-46.

30. Fiser DH, Tilford JM, Roberson PK (2000) Relationship of illness severity and length of stay to functional outcomes in the pediatric intensive care unit: A multi-institutional study. Crit Care Med 28: 1173-1179.

31. Ebrahim S, Singh S, Hutchison JS, et al. (2013) Adaptive behavior, functional outcomes, and quality of life outcomes of children requiring urgent ICU admission. Pediatr Crit Care Med 14: 10-18. 